\title{
彩色摄像机颜色分辨力测量
}

\author{
徐记伟 ${ }^{1}$, 杜 仙 $^{2}$, 方海棠 ${ }^{3}$, 邢冀川 ${ }^{1}$
}

(1. 北京理工大学 光电学院, 北京 $100081 ; 2$. 国网宜昌供电公司信息通信分公司, 湖北 宜昌 443000;

3. 塔里木油田公司信息与通讯技术中心，新疆 库尔勒 841000）

\begin{abstract}
摘要: 为实现对彩色摄像机颜色分辨力测试, 设计了通过双离散颜色源、反射式准直光学系统和四杆 靶靶标模块组成的测试装置。双离散颜色源分别产生经过标定的前景和背景颜色光束, 经过准直光学 系统, 最终合成四杆靶测试靶标图像。该系统在测量过程分别获取各频率下从靶标图像中提取的目标 靶标并对其进行色差计算, 通过图像算法对测试靶标进行判断和识别, 获得彩色摄像机的最小可分辨 色差 (minimum resolvable $E$ difference, MRED) 和最小可探测色差（minimum detectable $E$ difference, MDED），实现对彩色摄像机颜色分辨力的客观检测。
\end{abstract}

关键字: 彩色摄像机; 颜色分辨力; 四杆靶测试靶标

中图分类号：TN948.41 文献标识码：A 文章编号：1001-8891(2020)05-0468-05

\section{Measurement of Color Resolution for Color Cameras}

\author{
XU Jiwei ${ }^{1}$, DU Xian ${ }^{2}$, FANG HaiTang ${ }^{3}$, XING Jichuan ${ }^{1}$ \\ (1. Beijing Institute of Technology, School of Optics and Photonics, Beijing 100081, China;
}

2. State Grid Yichang Power Supply Company Information \&Telecommunication Branch, Yichang 433000, China;

3. Tarim Oilfield Company Information and Communication Technology Corporation, Korla 841000, China)

\begin{abstract}
In order to calibrate the color resolution of a color camera, an experimental prototype is designed which consists of a double discrete color source, a reflective collimating optical system and four-bar target panel. The dual discrete color sources respectively produce a foreground and background color beam passing through a collimating optical system, and then form a four-bar synthetic image on the color CCD. In the experiment, we extracts the stripe from the target image at each frequency and calculates the color difference of each pixel. Then, we use the criterion to mark the detectable pixels and define the minimum resolvable $E$ difference (MRED) and the minimum detectable $E$ difference (MDED) as two indexes to objectively evaluate the color resolution of the color camera.
\end{abstract}

Key words: color camera, color resolution, four-bar target test target

\section{0 引言}

在视觉识别、艺术再现和军事目标探测领域, 彩 色摄像机相比于单色成像系统, 能够从空间场景中获 取更丰富的频率信息。然而彩色摄像机也面临图像颜 色失真、不均匀性等问题, 严重降低了彩色摄像机的 空间分辨力。到目前为止, 现有对成像传感器分辨力 的研究大部分集中在 “黑白” 问题，只有很少部分涉 及到颜色分辨力的测量 ${ }^{[1]}$, 为了获得彩色摄像机对两 种颜色差异的分辨能力, 因此需要对彩色摄像机颜色 分辨力进行精确地测量。
典型的颜色精度测试是通过颜色测试卡来进行 颜色测试，然而这种方法工作量大，而且无法测试不 同空间频率的色差。文献[2]和文献[3]中使用两路能产 生特定颜色共轭像的光源及平行光管组成的颜色分 辨力测量系统都需要由人来对彩色摄像机颜色分辨 力进行主观判断。本文提出了通过标定的颜色光源产 生双路控制四杆靶标图像, 并结合图像算法对靶标图 像中的目标进行探测和识别的颜色分辨力测量系统 将会更加客观、有效地实现颜色精度和分辨力的测 量。 


\section{1 彩色摄像机颜色分辨力测试系统}

本装置由一个双离散的颜色光源和反射式目标 靶组成的单个光学系统, 产生双路颜色可调的单色光 照射测试靶标, 通过测试靶标来填充被测彩色图像传 感器, 由此对彩色摄像机的颜色分辨力进行测试。其 中每一个颜色源都处于闭环控制状态, 整个系统的输 出被精确地校准。实验装置原理图如图 1 所示。

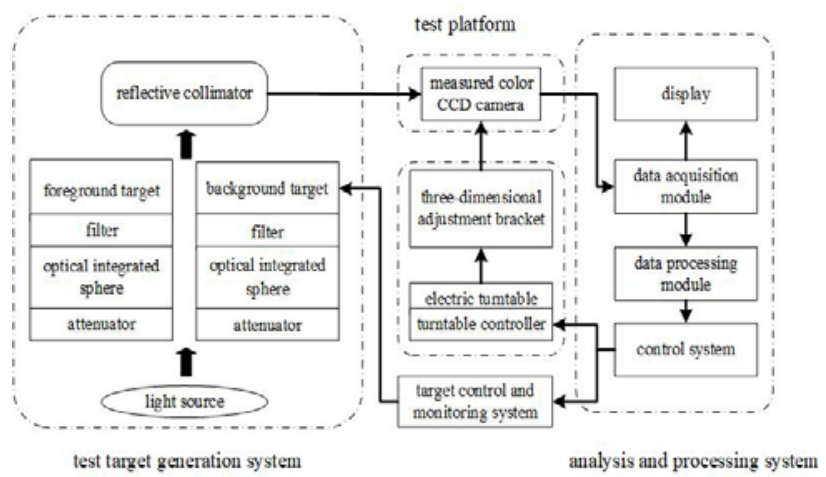

图 1 彩色摄像机颜色分辨力实验装置原理图

Fig.1 Schematic diagram of color camera color resolution experimental device

颜色光源是通过两组双积分球 ${ }^{[4]}$, 来产生颜色可 调的双路颜色光。双积分球装置主要由主积分球、次 积分球和滤光片组等组成。主积分球和次积分球主要 是为了在滤光片上得到均匀光和消除滤光片所引起 光源的不均匀性; 滤光片通过 3 个可独立运动的滤光 片在次积分球内产生任意的颜色光。为了对输出的颜 色光进行精确地控制, 每一个双积分球颜色源上都有 两个光亮度传感器和光谱传感器形成闭环控制系统, 分别对光源的亮度和光谱特性进行精确控制。双积分 球光源结构如图 2 所示。

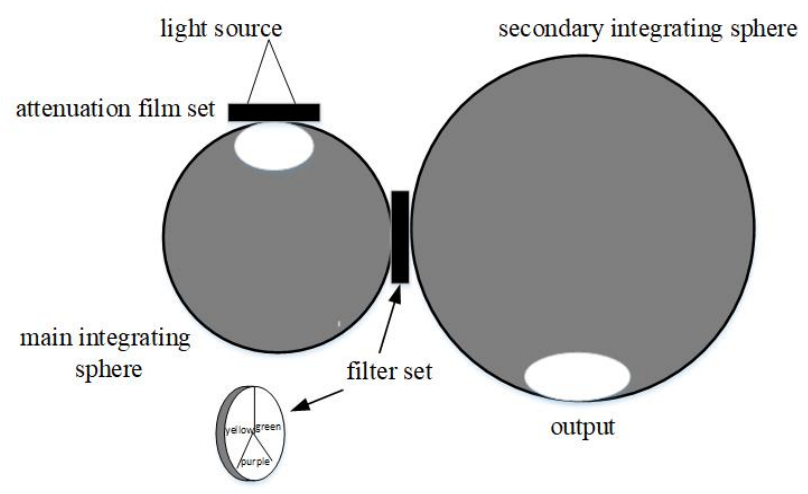

图 2 双积分球颜色源系统结构

Fig.2 Double integrating sphere color source system structure

测试靶标是进行彩色摄像机颜色分辨力测试的 核心部件, 双路颜色光源分别照射测试靶标, 通过装 置中反射式光学系统将靶标图像投射在被测彩色图 像传感器上。为了实现双路颜色测试图像, 测试靶标
是成对设计的, 分为前景靶标和背景靶标。测试靶标 合成如图 3 所示。

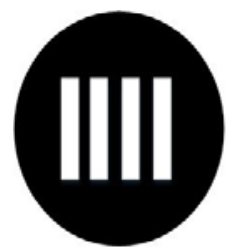

foreground target

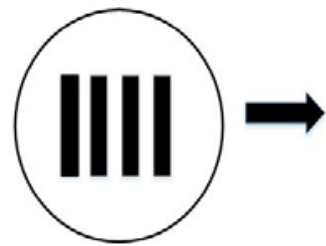

background target

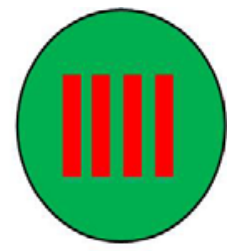

test target
图 3 测试靶标合成图

Fig.3 Test target synthesis diagram

为了提高颜色光的保真度, 系统采用了反射式光 学系统 ${ }^{[5]}$ 。传统的反射型准直器可以有一个折返镜和 一个离轴抛物面组成, 但是本系统要求有两路颜色靶 标图样合成一路测试靶标图像, 因此在光路中加入了 一个分光棱镜用于在光谱和空间上组合前景通道和 背景通道。光学系统原理图如图 4 所示。

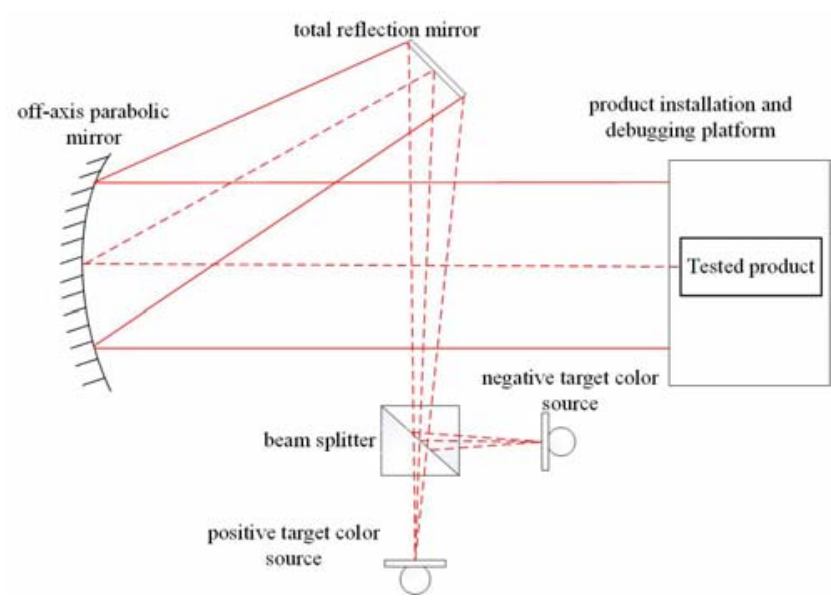

图 4 光学系统原理图

Fig.4 Optical system schematic

\section{2 彩色图像的特征提取与色差计算}

\section{1 目标特征提取}

通过测试靶标图像客观地测试彩色摄像机颜色 分辨力的过程中, 需要对测试靶标图像进行预处理。 在人主观测量彩色摄像机的颜色分辨力时, 由于人眼 观察图像具有一定的积分效应 ${ }^{[6]}$, 一定程度上消除了 部分随机噪声, 因此在预处理时不进行过多的图像滤 除噪声和改善图像信噪比。仅仅对彩色摄像机采集的 靶板图像在 $0.1 \mathrm{~s}$ 内进行帧平均处理去除部分随机噪 声, 同时避免了图像条纹特征的失真 ${ }^{[7]}$ 。

要从图像中提取颜色条纹特征首先要确定四杆 靶的位置, 选取颜色差异较大的靶标图像, 通过自适 应边缘检测和图像分割算法分别对测试靶标的感兴 趣区域和靶杆位置区域进行处理。由于在测量过程中 靶板相对于摄像机处于静止状态, 将此区域作为一个 
定值。测试靶标图像处理流程图, 如图 5 所示。

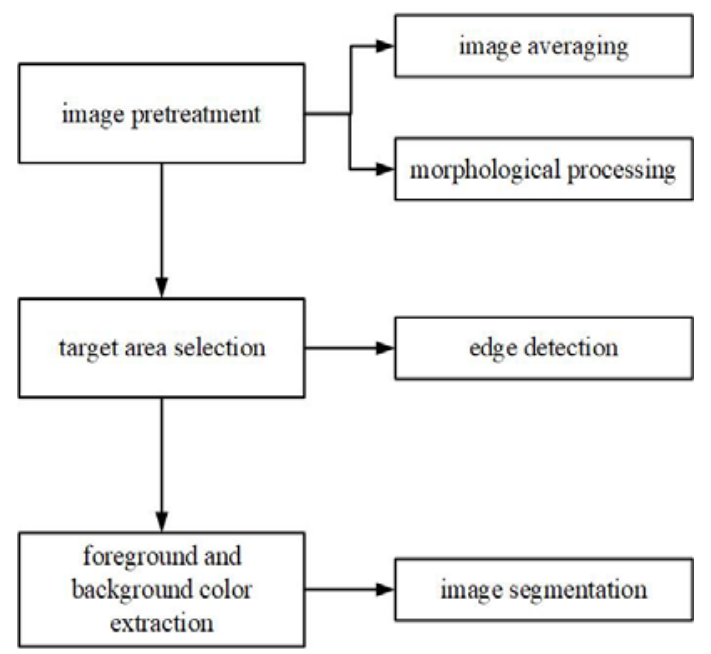

图 5 测试靶标图像处理流程图

Fig.5 Test target image processing flowchart

\section{2 图像色差的计算}

图像色差进行计算是在三维 CIE $\mathrm{L}^{*} \mathrm{a} * \mathrm{~b}$ *均匀色 度空间中进行的。CIELAB 均匀颜色空间以等距性好、 色差分辨力高 ${ }^{[2]}$ 以及更加逼近人类的视觉感知, 已经 作为国际通用的测色标准。如图 6 所示, $\mathrm{L}^{*} \mathrm{a} * \mathrm{~b} *$ 色度 空间立体图。

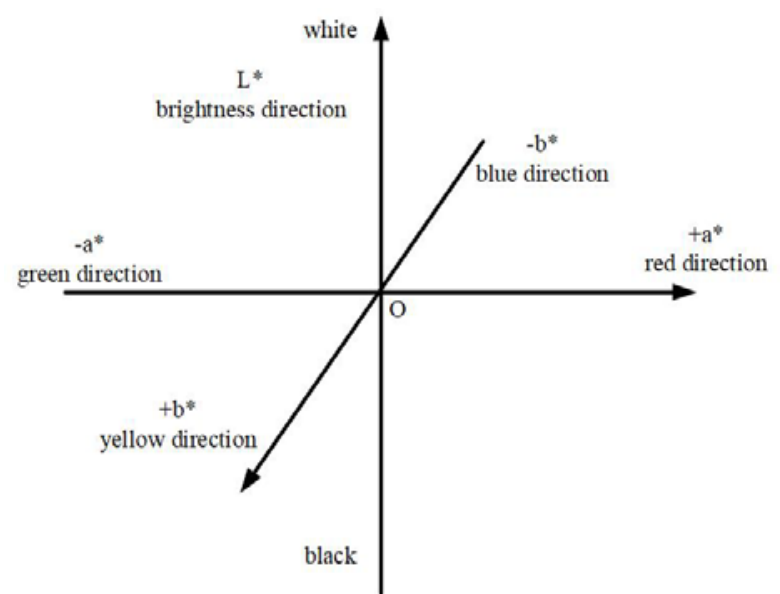

图 $6 \mathrm{~L} * \mathrm{a} * \mathrm{~b} *$ 色度空间立体图, $\mathrm{L} *$ 表示颜色的明度, $\mathrm{a} *$ 正值表 示偏红, 负值表示偏绿; b*正值表示偏黄, 负值表示偏 蓝

Fig.6 L*a*b* chromaticity space stereogram, $L *$ represents the lightness of the color, $a^{*}$ positive value means reddish, negative value means greenish; $b^{*}$ positive value means yellowish, negative value means blueish

2.2.1 颜色空间的转换

假设彩色摄像机的颜色空间为 $\mathrm{sRGB}^{[8]}$ ，而 $\mathrm{sRGB}$ 和 CIELAB 颜色空间是不能直接转化的, 需要先从 sRGB 颜色空间转化为 CIEXYZ 颜色空间, 在经过 CIEXYZ 颜色空间变换到 CIELAB 颜色空间, 转化程 序如图 $7^{[8]}$ 所示。由 sRGB 转化为 CIELAB 可以通过
一个 $3 \times 3$ 的矩阵转换来实现 ${ }^{[9]}$ :

$$
\left(\begin{array}{l}
X \\
Y \\
Z
\end{array}\right)=\Phi_{\mathrm{sRGB} \rightarrow X Y Z}\left(\begin{array}{l}
R \\
G \\
B
\end{array}\right)=100\left(\begin{array}{lll}
0.4124 & 0.3576 & 0.1805 \\
0.2126 & 0.1752 & 0.0722 \\
0.0193 & 0.1192 & 0.9505
\end{array}\right)\left(\begin{array}{l}
R \\
G \\
B
\end{array}\right)
$$

由 CIEXYZ 转化为 CIELAB 的转化公式 ${ }^{[9]}$ 如下: 明度:

$$
L^{*}=166\left(\frac{Y}{Y_{n}}\right)^{1 / 3}-16 \quad \frac{Y}{Y_{n}}>0.008856
$$

色度:

$$
\left\{\begin{array}{l}
a^{*}=500\left[\left(\frac{X}{X_{n}}\right)^{1 / 3}-\left(\frac{Y}{Y_{n}}\right)^{1 / 3}\right] \\
b^{*}=200\left[\left(\frac{Y}{Y_{n}}\right)^{1 / 3}-\left(\frac{Z}{Z_{n}}\right)^{1 / 3}\right] \\
\frac{X}{X_{n}}, \frac{Y}{Y_{n}}, \frac{Z}{Z_{n}}>0.008856
\end{array}\right.
$$

式中: $X_{n} 、 Y_{n} 、 Z_{n}$ 为照明光源的三刺激值所对应标准 照明体的三刺激值。

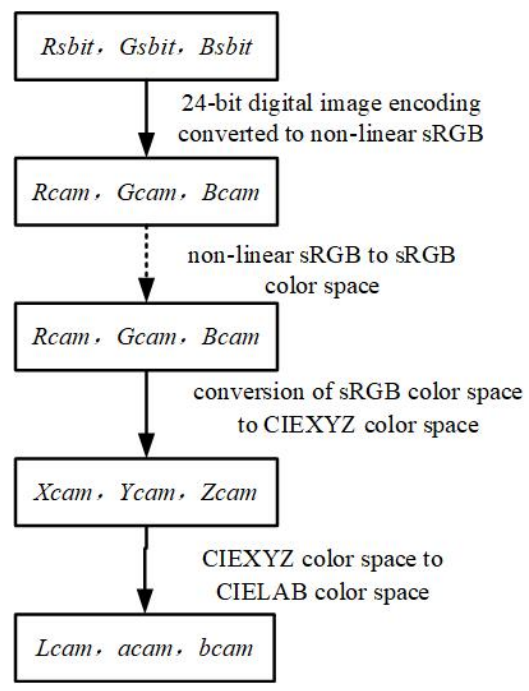

图 7 颜色空间转化程序处理流程图

Fig.7 Color space conversion program processing flowchart 2.2.2 图像色差计算

彩色摄像机采集的测试靶标图像采用的是双色 四杆靶图像，可以把前景色和背景色作为感兴趣区域 分开处理。在 CIELAB 颜色空间中, $L^{*} 、 a^{*} 、 b^{*}$ 三个 分量对总体平均色差的贡献是相互独立的，因此可以 通过对前景靶标区域和背景靶标区域分别求 3 个分量 的算术平均值 ${ }^{[10]}$ : 


$$
\overline{L^{*}}=\frac{\sum L^{*}}{n} \quad \overline{a^{*}}=\frac{\sum a^{*}}{n} \quad \overline{b^{*}}=\frac{\sum b^{*}}{n}
$$

可以求得前景靶标区域和背景靶标区域在 CIELAB 颜色空间的色度坐标为 $\left(\overline{L_{1}^{*}}, \overline{a_{1}^{*}}, \overline{b_{1}^{*}}\right)$ 和 $\left(\overline{L_{2}^{*}}, \overline{a_{2}^{*}}, \overline{b_{2}^{*}}\right)$ 。色差可用颜色空间中的几何距离 ${ }^{[11]}$ 来表 示:

$$
\Delta E=\sqrt{\left(\overline{L_{1}^{*}}-\overline{L_{2}^{*}}\right)^{2}+\left(\overline{a_{1}^{*}}-\overline{a_{2}^{*}}\right)^{2}+\left(\overline{b_{1}^{*}}-\overline{b_{2}^{*}}\right)^{2}}
$$

对彩色摄像机颜色分辨力进行测量就需要色差阈值 作为对测试图像判定的依据, 当所测量的色差小于该 阈值就无法分辨, 反之可以分辨; 在彩色电视系统中 两种颜色恰巧刚好不能区分的色差值 ${ }^{[12]}$ ( just noticeable color difference, JNCD) 为5 NBS (Dalton,

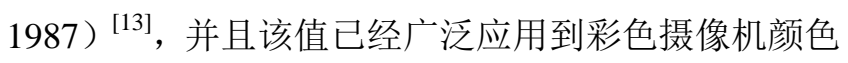
检测和校正中 ${ }^{[14]}$ 。因此取该值作为设备的色差阈值。 测试图像的可分辨区域的划分可通过式(6)来描述:

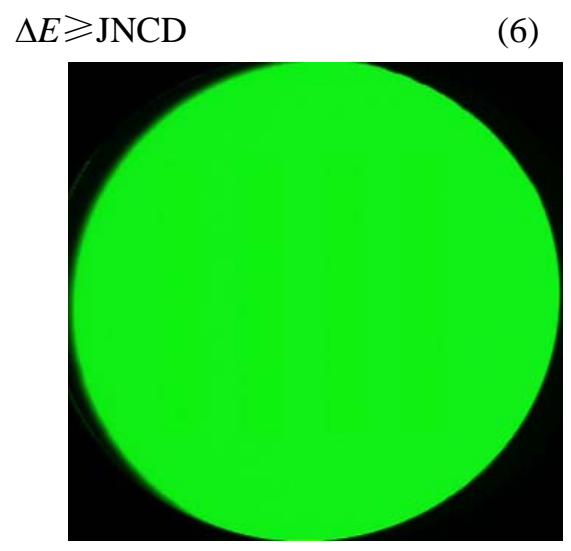

(a) 原始图像

(a) Original image

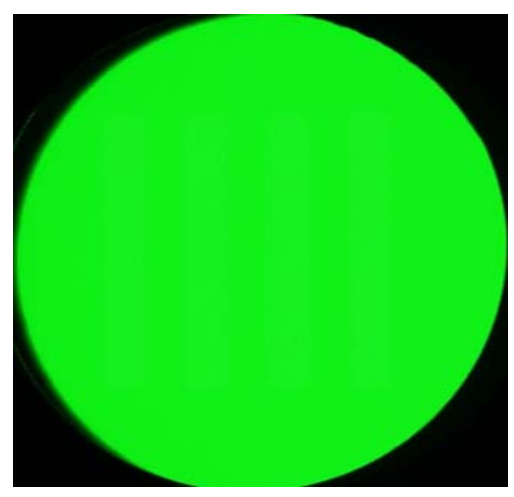

(c) 原始图像

(c) Original image

(b) MDED target recognition test image

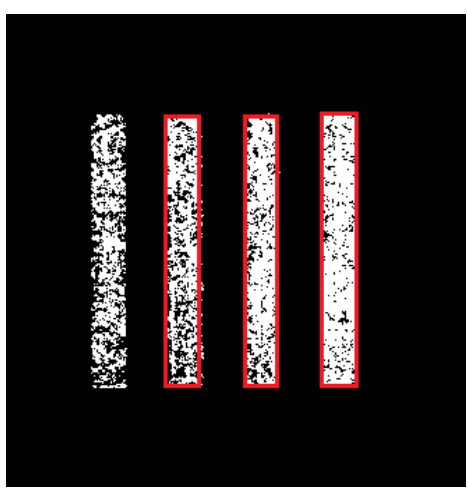

(d) MRED 目标识别测试图像

(d) MRED target recognition test image

图 8 彩色摄像机的最小可探测色差测试图像和最小可分辨色差测试图像

Fig.8 Test image of minimum detectable E difference and minimum resolvable E difference of color camera 


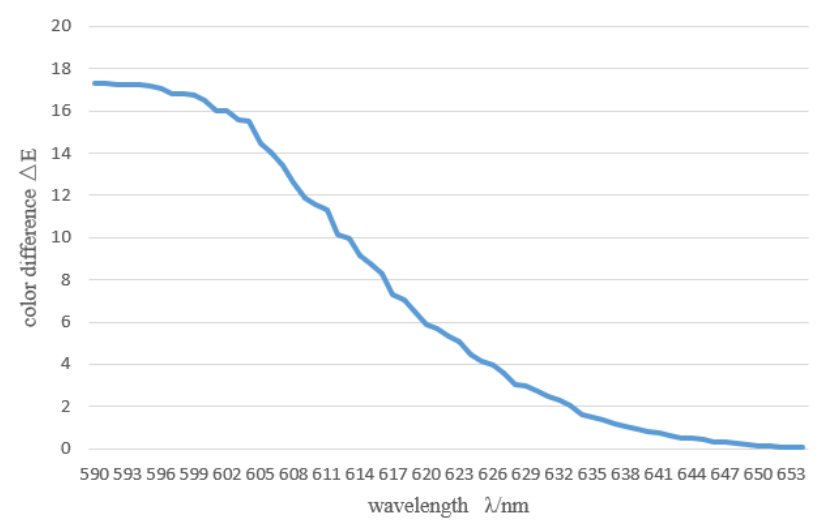

图 9 颜色分辨力测试装置从 $590 \mathrm{~nm} \sim 653 \mathrm{~nm}$ 的色差变化曲线

Fig.9 Color difference test curve of color resolution test device from $590 \mathrm{~nm}-653 \mathrm{~nm}$

在此过程中, 用被测彩色摄像机连续采集 50 幅 图像, 对获取的目标图像数据预处理 (图像平均运算) 后, 作为对彩色摄像颜色分辨力进行测试的颜色样 本。通过图 5 中所示中靶板前景和背景目标提取算法, 对获取四杆靶图像中的区域目标按照图 7 所示的颜色 空间转换算法变换到 Lab 颜色空间, 在经过公式(4) 和公式(5)计算前景目标和背景目标的色差。MRED 和 MDED 可通过公式(6)计算能够探测到的靶杆像素个 数并结合 JNCD 判别标准对测试靶杆进行分辨。重复 上面的测试步骤, 只需要更换分辨力靶板的便可以计 算出不同分辨力下的最小可分辨色差和最小可探测 色差。测试结果如图 10 所示。

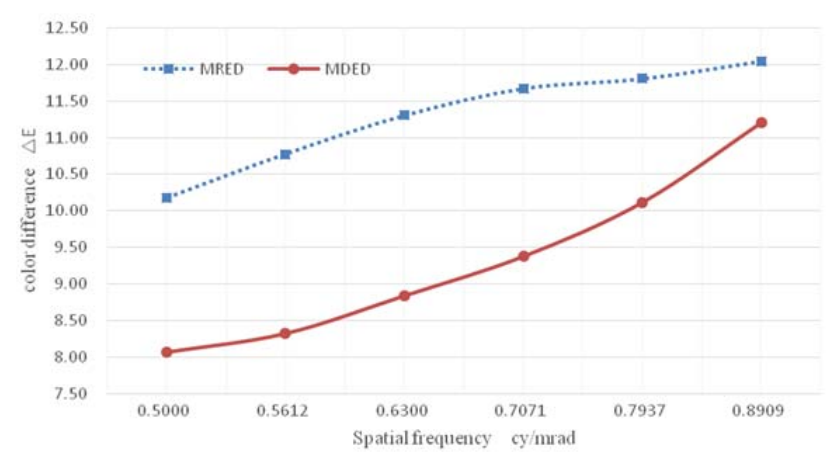

图 10 彩色摄像机颜色分辨力测试曲线

Fig.10 Color camera color resolution test curve

由图 10 可知, 随着空间频率越大, 最小可探测 和最小可分辨色差也逐渐增加, 这一规律符合客观事 实。但是其增加的幅度不大, 在 $3 \mathrm{NBS}$ 左右, 该值小 于摄像机可以识别的最小色差 $5 \mathrm{NBS}$, 可以忽略不计。 因此, 在某一种颜色波段范围内 (前景色的亮度、饱 和度不变, 只改变前景波长的情况下), 我们可以通 过该方法测出各种颜色的最小可探测和最小可分辨 色差。

\section{4 总结}

本文介绍了使用双色图样测试靶标结合反射式 光学系统组成的彩色摄像机颜色分辨力测试系统, 该 系统通过图像处理算法客观的测量了不同分辨力下 彩色摄像机的 MDED 和 MRED。从测试结果分析, 我们可以测量和标定彩色摄像机对颜色的分辨能力, 尤其对在特殊场合或者在某一特定颜色应用的彩色 摄像机的检测提供了客观可行的方法, 而且具有方 便、快速、准确等特点。该系统也可以应用在对商业 摄像机、彩色印染检测摄像机、智能机器人中的人眼 仿生相机和彩色医疗设备等颜色分辨力检测方面。

\section{参考文献:}

[1] SONG Yuehong, XU Junqing. Digital Camera Color Resolution Test Based on Knife-Edge Method[J]. Applied Mechanics and Materials, 2013, 469: 246-250.

[2] 连礼泉. 色差分辨力测量 [C]//第十三届全国光学测试学术讨论会论 文, 2010: 202.

LIAN Liquan. The measurement of color resolution[C]//Proceedings of the $13^{\text {th }}$ National Symposium on optical measurement, 2010: 202.

[3] Jason A Mazzetta, Jason A Mazzetta, Stephen D Scopatz, et al. Relative color delineation testing of visible camera systems[C]/Proc. SPIE, 2008: 694116.

[4] 荆其诚, 焦书兰, 喻柏林, 等. 色度学[M]. 北京: 科学出版社, 1979. JING Qicheng, JIAO Shulan, YU Bailin, et al. Chromaticity[M]. Beijing: Science Press, 1979

[5] 顾小超, 胡雯雯, 黄丽俐, 等. 一种离轴抛物面准直光管的设计 [J]. 激 光与红外, 2008, 38(5): 472-473.

GUO Xiaochao, HU Wenwen, HUANG Lili, et al. Design of the Off-axis Collimator[J]. Laser \& Infrared, 2008, 38(5): 472-473

[6] 董伟, 郭䞨, 张伟. MRTD 客观测量中四杆靶图像的特征提取[J]. 激光 与红外, 2002, 32(2): 104-106.

DONG Wei, GUO Ting, ZHANG Wei. The Extraction of Character of Four Bacilliform Target Image in the Objective Measurement of MRTD[J]. LASER \& INFRARED, 2002, 32(2): 104-106.

[7] 王东伟, 张旭生, 何川, 等. 红外热像仪最小可辩温差客观测评技术 [J]. 红外与激光工程, 2010, 39(4): 611-614.

WANG Dongwei, ZHANG Xusheng, HE Chuan, et al. Objective evaluation method for MRTD of IR themal imager[J]. Infrared and Laser Engineering, 2010, 39(4): 611-614.

[8] Renee Charriere, Mathieu Hebert, Alain Trémeau, et al. Color calibration of an RGB camera mounted in front of a microscope with strong color distortion[J]. Applied optics, 2013, 52(21): 5262-5271.

[9] Yves Vander Haeghen, et al. An Iamging System with Calibrated Color Image Acquisition for Use in Dermatology[J]. IEEE Transactions on Medical Imaging, 2000, 19(7): 722-730.

[10] 孙建明, 周世生, 周利国. 基于 CIEDE2000 色差公式的印刷品色差 检测研究[J]. 机械科学与技术, 2011, 30(4): 582-585.

SUN Jianming, ZHOU Shisheng, ZHOU Liguo. Detection of the Printing Color Difference Based on CIEDE2000 Color-difference Formula[J]. Mechanical Science and Technology for Aerospace Engineering, 2011, 30(4): 582-585.

[11] 刘浩学, 黄敏, 武兵, 等. 彩色数字图像色差计算方法的研究 [J]. 光 学学报, 2012, 32(9): 1-9.

LIU Haoxue, HUANG Min, WU Bing, et al. Study of Difference Evaluation in Color Digital Images[J]. Acta Optica Sinica, 2012, 32(9): $1-9$.

[12] LIU Kuocheng. An Improvement of Just Noticeable Color Difference Estimation[C]//Proceedings of the Federated Conference on Computer Scienceand Information Systems, 2016, 8: 803-806.

[13] N J C Strachan, P Nesvadba, et al. Calibration of a video camera digitizing in the CIE $\mathrm{L}^{*} \mathrm{u}^{*} \mathrm{o}^{*}$ colour space[J]. Pattern Recognition Letters, 1990, 11(11): 771-777.

[14] Montse Corbalán, Marı́a S. Millán, Marı́a J. Yzuel. Color measurement in standard CIELAB coordinates using a 3CCD camera: correction for the influence of the light source[J]. Society of Photo-Optical Instrumentation Engineers, 2000, 39(6): 1470-1476. 